\title{
Standardization of the NEO-PI-3 in the Greek general population
}

\author{
Konstantinos N Fountoulakis ${ }^{*}$, Melina Siamouli, Stefania Moysidou², Eleonora Pantoula², Katerina Moutou², \\ Panagiotis Panagiotidis ${ }^{3}$, Marina Kemeridou², Eirini Mavridou ${ }^{2}$, Efimia Loli ${ }^{4}$, Elena Batsiari ${ }^{5}$, Antonio Preti ${ }^{6}$, \\ Leonardo Tondo ${ }^{7,8}$, Xenia Gonda ${ }^{9}$, Nisreen Mobayed ${ }^{10}$, Kareen Akiskal $^{11}$, Hagop Akiskal ${ }^{12}$, Paul Costa ${ }^{13}$ \\ and Robert McCrae ${ }^{14}$
}

\begin{abstract}
Background: The revised NEO Personality Inventory (NEO-PI-3) includes 240 items corresponding to the Big Five personality traits (Extraversion, Agreeableness, Conscientiousness, Neuroticism, and Openness to Experience) and subordinate dimensions (facets). It is suitable for use with adolescents and adults (12 years or older). The aim of the current study was to validate the Greek translation of the NEO-PI-3 in the general Greek population.

Material and methods: The study sample included 734 subjects from the general Greek population of whom 59.4\% were females and $40.6 \%$ males aged $40.80 \pm 11.48$. The NEO-PI-3 was translated into Greek and back-translated into English, and the accuracy of the translation was confirmed and established. The statistical analysis included descriptive statistics, confirmatory factorial analysis (CFA), the calculation of Cronbach's alpha, and the calculation of Pearson product-moment correlations. Sociodemographics groups were compared by ANOVA.

Results: Most facets had Cronbach's alpha above 0.60. Confirmatory factor analysis showed acceptable loading of the facets on their own hypothesized factors and very good estimations of Cronbach's alphas for the hypothesized factors, so it was partially supportive of the five-factor structure of the NEO-PI-3.The factors extracted with Procrustes rotation analysis can be considered reasonably homologous to the factors of the American normative sample. Correlations between dimensions were as expected and similar to those reported in the literature.

Discussion: The literature suggests that overall, the psychometric properties of NEO-PI-3 scales have been found to generalize across ages, cultures, and methods of measurement. In accord with this, the results of the current study confirm the reliability of the Greek translation and adaptation of the NEO-PI-3. The inventory has comparable psychometric properties in its Greek version in comparison to the original and other national translations, and it is suitable for clinical as well as research use.
\end{abstract}

Keywords: Five-factor personality inventory, NEO-PI-3, Standardization, Psychometrics

\section{Introduction}

The NEO Inventories were developed by Paul T. Costa, Jr. and Robert R. McCrae. Because it assessed Neuroticism, Extraversion, and Openness to experience, its original version, developed in 1978, is known as the NEO inventory (NEO-I). The NEO-I measured only three of the Big Five personality traits [1] and was subsequently revised in 1985 to include all five traits under the new title 'NEO

\footnotetext{
*Correspondence: kfount@med.auth.gr

${ }^{1}$ Third Department of Psychiatry, School of Medicine, Aristotle University of Thessaloniki, Thessaloniki, Greece

Full list of author information is available at the end of the article
}

Personality Inventory (NEO-PI).' It was further refined as the NEO-PI-R [2]. Its latest version is the NEO-PI-3 [3].

The NEO-PI-3 includes 240 items corresponding to the Big Five personality traits (Extraversion, Agreeableness, Conscientiousness, Neuroticism, and Openness to xperience) and subordinate dimensions (facets). It is suitable for use with adolescents and adults (12 years or older). Item responses are made on a five-point scale, ranging from 'strongly disagree' to 'strongly agree'. Electronic and print forms of the inventories are available. Administration of the full version of the NEO-PI-3 takes between 
30 and $40 \mathrm{~min}$. Assessment should not be evaluated if there are more than 40 items missing.

The aim of the current study is to validate the Greek translation of the NEO-PI-3 in the general Greek population.

\section{Material and methods}

The study sample included 734 subjects from the general Greek population (436 females, 59.4\%; 298 males, 40.6\%). Their mean age was $40.80 \pm 11.48$ years (range 25-67 years): $39.43 \pm 10.87$ years (range $25-65$ years) for females and $42.82 \pm 12.06$ years (range 25-67 years) for males.

The NEO-PI-3 was translated into Greek by KNF and back-translated into English by two other authors (MS and $\mathrm{KM}$ ). The originators of the instrument and KNF verified the accuracy of the translation and its conformity to the original version. Discrepancies were discussed until an agreement was reached. This final version was then refined to ensure it is easily understandable.

\section{Statistical analysis}

All data were coded and analyzed using the Statistical Package for Social Sciences (SPSS) version 20 (SPSS Inc., Chicago, IL, USA). All tests were two-tailed. According to the Bayesian interpretations, the chance of replication in future studies is low for $p$ values between 0.05 and 0.01 , moderate for $p$ values between 0.01 and 0.001 , and high for $p<0.001$ [4].

First, descriptive statistics (means, standard deviations, and frequency tables) were calculated for the items and subscales proposed by Costa and McCrae [5]. Second, with the aim of studying the structure of the NEO-PI-3, a confirmatory factorial analysis (CFA), was conducted at the facets level (see below); a targeted rotation of principal components was also evaluated using congruence coefficients with the American normative sample.

Scale reliability was measured by Cronbach's alpha. For group comparisons, reliability values of 0.7 are considered satisfactory while subscales values approximately 0.6 are considered acceptable [6]. However, it has been argued that internal consistency is less important than retest reliability [7].

The Pearson product-moment correlation method was used to determine the presence or absence of variable correlation. This method was chosen due to its robustness with regards to normality assumptions and for its simple interpretability. For Pearson's $r$, the suggested threshold for effect sizes were $r=0.10=$ small effect, $r=$ $0.24=$ medium effect, and $r=0.37=$ large effect [8].

Sociodemographics groups were compared by ANOVA.

\section{Confirmatory factorial analysis}

CFA was carried out with the lavaan package [9] running in $\mathrm{R}[10]$. The lavaan package has been shown to
Table 1 Composition of the study sample in terms of gender and age in comparison to the general population according to the Greek National Statistics Service for 2009

\begin{tabular}{lll}
\hline Age group & $\begin{array}{l}\text { Greek population } \\
\text { (approximation for 2009) }\end{array}$ & Study sample \\
\hline Total population & $11,282,751$ & 734 \\
Males vs. females & $48 \%$ vs. 52\% & $40.6 \%$ vs. 59.4\% \\
25-29 years old & $11.02 \%$ & $25.81 \%$ \\
29-34 years old & $11.31 \%$ & $12.90 \%$ \\
34-39 years old & $10.00 \%$ & $15.44 \%$ \\
$40-44$ years old & $10.00 \%$ & $13.13 \%$ \\
$44-49$ years old & $9.21 \%$ & $10.60 \%$ \\
$50-54$ years old & $8.92 \%$ & $10.14 \%$ \\
$55-59$ years old & $6.83 \%$ & $8.29 \%$ \\
$60-64$ years old & $7.09 \%$ & $3.69 \%$ \\
\hline
\end{tabular}

generate the same results as other software packages [11]. Mardia's kurtosis was used to check for multivariate non-normality: Mardia's kurtosis $=1,194, z=30.78$, $p<.0001$.

Maximum likelihood estimation with robust standard errors and the Satorra-Bentler scaled test statistic were used to test CFA models; this method was chosen because it was unlikely to be affected by deviation from normality in data [12]. Chi square is the traditional fit index used to evaluate an overall model as it assesses the magnitude of discrepancy between the sample and the fitted covariance matrices [13]. However, the use of the chi square test to assess this model fit was found unsatisfactory for a number of reasons [14], including its sensitivity to sample size. The ratio of chi square to the degrees of freedom $(d f)$ was calculated, with ratios larger than 3 indicating poor fit [15]. Additional parameters for fit estimation were the following: the comparative fit index (CFI), the root mean square error of approximation (RMSEA), and the standardized root mean square residual (SRMR). RMSEA values of 0.08 or lower, SRMR

\section{Table 2 Occupation characteristics of the study sample}

\begin{tabular}{lll}
\hline & Count & Percentage \\
\hline He/she used to work but is currently unemployed & 0 & 0.00 \\
He/she never worked and neither does now & 0 & 0.00 \\
Clerk (civil or private) & 338 & 63.41 \\
Free professional (tradesman, craftsman) & 62 & 11.63 \\
Doctor, lawyer, engineer, priest, teacher, etc. & 75 & 14.07 \\
Student (college or university) & 12 & 2.25 \\
Blue collar worker (construction worker, farmer) & 26 & 4.88 \\
Housewife & 20 & 3.75 \\
Total & 533 & 100.00 \\
\hline
\end{tabular}


values of 0.09 or lower, and CFI values of 0.90 or higher are considered acceptable $[13,16]$.

Two models were tested, a rather unlikely, unidimensional model, which assumes that all facets load on a single factor, and the a priori expected five-factor model, in which all facets were linked to its own latent factor only, the so-called simple structure $[17,18]$. The more complex models were not tested because they are based on cross-loading (as well as several cross-loadings), which prevents a clear attribution of the predictor to the latent variable it is expected to measure. As a matter of fact, it has been found that increasing the measure's complexity to comply with the CFA standard led to a reduced convergent and discriminant validity [17].

When CFA failed to reach fit, the orthogonal Procrustes rotation was proposed as a method to test the replicability of the NEO-PI-3 personality factors [18-20]. A dedicated script running in SPSS of the program that performs the

Table 3 Mean values for the domains and the facets of the Greek NEO-PI-3

\begin{tabular}{|c|c|c|c|c|c|c|}
\hline NEO-PI-3 facet & Mean & SD & Skewness & Kurtosis & Cronbach's alpha $(95 \% \mathrm{Cl})$ & $\begin{array}{l}\text { Cronbach's alpha } \\
\text { (United States) }\end{array}$ \\
\hline Neuroticism & 89.06 & 19.59 & 0.05 & 0.19 & & \\
\hline N1: Anxiety & 17.39 & 5.05 & -0.14 & -0.14 & $0.724(0.693-0.753)$ & 0.72 \\
\hline N2: Hostility & 14.25 & 4.15 & 0.24 & 0.45 & $0.582(0.534-0.626)$ & 0.69 \\
\hline N3: Depression & 14.07 & 4.92 & 0.23 & -0.15 & $0.708(0.674-0.739)$ & 0.80 \\
\hline N4: Self-consciousness & 15.43 & 3.99 & 0.21 & 0.05 & $0.467(0.406-0.523)$ & 0.66 \\
\hline N5: Impulsivity & 15.48 & 3.87 & 0.13 & -0.07 & $0.425(0.360-0.486)$ & 0.63 \\
\hline N6: Vulnerability & 12.44 & 4.45 & 0.26 & 0.27 & $0.685(0.649-0.718)$ & 0.70 \\
\hline Extraversion & 108.68 & 15.97 & -0.03 & -0.15 & & \\
\hline E1: Warmth & 21.10 & 4.10 & -0.58 & 1.01 & $0.648(0.608-0.685)$ & 0.73 \\
\hline E2: Gregariousness & 18.17 & 4.43 & -0.08 & -0.13 & $0.613(0.569-0.654)$ & 0.77 \\
\hline E3: Assertiveness & 15.90 & 4.14 & 0.18 & 0.05 & $0.589(0.543-0.633)$ & 0.76 \\
\hline E4: Activity & 18.64 & 3.79 & 0.10 & 0.02 & $0.501(0.445-0.554)$ & 0.61 \\
\hline E5: Excitement-seeking & 15.66 & 3.96 & 0.04 & 0.13 & $0.445(0.382-0.503)$ & 0.63 \\
\hline E6: Positive emotions & 19.21 & 4.30 & -0.28 & 0.28 & $0.636(0.594-0.674)$ & 0.77 \\
\hline Openness & 104.83 & 16.31 & 0.27 & 0.21 & & \\
\hline O1: Fantasy & 16.07 & 4.77 & 0.22 & -0.18 & $0.676(0.640-0.711)$ & 0.76 \\
\hline O2: Aesthetics & 18.02 & 5.15 & -0.07 & 0.01 & $0.729(0.698-0.757)$ & 0.79 \\
\hline O3: Feelings & 19.38 & 3.55 & 0.06 & -0.04 & $0.437(0.374-0.497)$ & 0.71 \\
\hline O4: Actions & 15.26 & 3.70 & 0.12 & 0.22 & $0.423(0.358-0.484)$ & 0.55 \\
\hline O5: Ideas & 16.88 & 4.83 & 0.07 & -0.05 & $0.694(0.659-0.726)$ & 0.79 \\
\hline O6: Values & 18.82 & 3.75 & 0.84 & 0.17 & $0.548(0.497-0.596)$ & 0.69 \\
\hline Agreeableness & 116.37 & 15.82 & -0.14 & 0.11 & & \\
\hline A1: Trust & 17.23 & 4.40 & -0.31 & -0.11 & $0.651(0.612-0.688)$ & 0.82 \\
\hline A2: Straightforwardness & 20.28 & 4.31 & -0.23 & 0.05 & $0.535(0.483-0.585)$ & 0.67 \\
\hline A3: Altruism & 22.39 & 4.07 & -0.59 & 0.59 & $0.669(0.631-0.704)$ & 0.74 \\
\hline A4: Compliance & 17.48 & 4.63 & -0.25 & -0.13 & $0.645(0.605-0.683)$ & 0.68 \\
\hline A5: Modesty & 17.82 & 3.37 & -0.08 & 0.05 & $0.301(0.221-375)$ & 0.70 \\
\hline A6: Tender-mindedness & 21.18 & 3.61 & -0.57 & 0.96 & $0.431(0.367-0.492)$ & 0.58 \\
\hline Conscientiousness & 121.60 & 19.22 & -0.16 & -0.09 & & \\
\hline C1: Competence & 19.86 & 3.70 & -0.14 & 0.29 & $0.511(0.455-0.562)$ & 0.65 \\
\hline C2: Order & 19.79 & 4.94 & -0.32 & -0.08 & $0.708(0.675-0.739)$ & 0.68 \\
\hline C3: Dutifulness & 22.79 & 4.34 & -066 & 0.85 & $0.655(0.616-0.692)$ & 0.64 \\
\hline C4: Achievement-striving & 20.44 & 4.16 & -0.30 & 0.05 & $0.636(0.595-0.674)$ & 0.73 \\
\hline C5: Self-discipline & 20.35 & 4.32 & -0.92 & 0.01 & $0.655(0.616-0.691)$ & 0.81 \\
\hline C6: Deliberation & 18.37 & 4.66 & -0.22 & -0.03 & $0.684(0.648-0.717)$ & 0.70 \\
\hline
\end{tabular}


orthogonal Procrustes rotation was used to execute the analysis (courtesy of Professor Robert R. McCrae).

According to a shared convention, factor loadings higher than 0.71 (accounting for $50 \%$ of variance or more) are considered excellent, $0.63(40 \%)$ very good, $0.55(30 \%)$ good values around 0.45 (20\%) fair, and values below 0.32 (10\% of variance) poor [21].

Congruence between potentially homologous factors across samples was evaluated using the coefficient of congruence (CC). The CC index ranges from -1.00 (perfect negative similarity) to 1.00 (perfect positive similarity), with zero indicating complete dissimilarity [22]. Reported thresholds for agreements between factors are as follows: very high $=0.90$ or above; high $=$ 0.80 to 0.89 ; and moderate $=0.70$ to 0.79 [23].

\section{Results}

The study sample was convenient and somewhat representative of the country's active population with some overrepresentation of younger ages and clerks (Tables 1 and 2).

Table 4 Confirmatory factor analysis of the facets of the Greek NEO-PI-3

\begin{tabular}{|c|c|c|c|c|c|}
\hline & Neuroticism & Extraversion & Openness to experience & Agreeableness & Conscientiousness \\
\hline N1: Anxiety & 0.724 & & & & \\
\hline N2: Hostility & 0.638 & & & & \\
\hline N3: Depression & 0.823 & & & & \\
\hline N4: Self-consciousness & 0.606 & & & & \\
\hline N5: Impulsivity & 0.466 & & & & \\
\hline N6: Vulnerability & 0.738 & & & & \\
\hline E1: Warmth & & 0.723 & & & \\
\hline E2: Gregariousness & & 0.571 & & & \\
\hline E3: Assertiveness & & 0.332 & & & \\
\hline E4: Activity & & 0.457 & & & \\
\hline E5: Excitement-seeking & & 0.415 & & & \\
\hline E6: Positive emotions & & 0.691 & & & \\
\hline O1: Fantasy & & & 0.480 & & \\
\hline O2: Aesthetics & & & 0.714 & & \\
\hline O3: Feelings & & & 0.567 & & \\
\hline O4: Actions & & & 0.422 & & \\
\hline O5: Ideas & & & 0.681 & & \\
\hline O6: Values & & & 0.435 & & \\
\hline A1: Trust & & & & 0.359 & \\
\hline A2: Straightforwardness & & & & 0.496 & \\
\hline A3: Altruism & & & & 0.874 & \\
\hline A4: Compliance & & & & 0.408 & \\
\hline A5: Modesty & & & & 0.282 & \\
\hline A6: Tender-mindedness & & & & 0.675 & \\
\hline C1: Competence & & & & & 0.665 \\
\hline C2: Order & & & & & 0.536 \\
\hline C3: Dutifulness & & & & & 0.713 \\
\hline C4: Achievement-striving & & & & & 0.706 \\
\hline C5: Self-discipline & & & & & 0.799 \\
\hline C6: Deliberation & & & & & 0.603 \\
\hline \multirow[t]{2}{*}{ Estimated Cronbach's alpha } & .830 & .717 & .723 & .719 & .826 \\
\hline & Robust chi square & Chi square/df & $\mathrm{CFI}$ & RMSEA $(90 \% \mathrm{Cl})$ & SRMR \\
\hline Expected & $p>.05$ & $<3$ & $>.900$ & $<.08(<.08)$ & $<.09$ \\
\hline Observed & $3,241.44, d f=395, p<.0001$ & 8 & 618 & $.099(.096-.102)$ & .119 \\
\hline
\end{tabular}


Internal consistency reliabilities and mean scores for the Greek NEO-PI-3 facets

Mean, standard deviation, skewness, kurtosis, and internal consistency scores (with 95\% confidence of interval) for the 30 NEO-PI-3 facets are shown in Table 3 .

Most facets exhibited Cronbach's alpha values above 0.60 , the accepted limit of internal consistency reliability for subscales. A few facets exhibited Cronbach's alpha values lower than 0.50 . Overall, the internal consistency reliability measures of the Greek translation were somewhat lower than those observed in the original American sample.

Skewness was always below [3.00] while kurtosis was always below [8.00], indicating that there was no univariate non-normality in the distribution of facet scores.

\section{Confirmatory factor analysis of the Greek NEO-PI-3}

The unidimensional model was rejected on the basis of the fit indexes: chi square $=4,975.31, d f=405, p<0.0001$; CFI $=$ 0.387; RMSEA = 0.124 (95\%CI: 0.121-0.127); SRMR = 0.137.

The a priori expected five-factor model had a better fit for all indexes (Table 4).

Overall, the fit was still poor. However, loading of the facets on their own hypothesized factors was acceptable, and the estimated Cronbach's alphas for the hypothesized factors were very good.

\section{Procrustes rotation analysis of the Greek NEO-PI-3}

The Procrustes rotation analysis revealed a good replication of the expected five-factor structure of the NEO-PI-3.

The loading of the facets on their own factors was good to excellent with few exceptions (Table 5).

Only a minority of facets also loaded on a different factor than their own with an absolute factor loading higher than 0.40 .

CC values for potentially homologous factors across samples were within high to very high interval. The extracted factors in the Greek sample can be considered reasonably homologous to their counterparts in the American normative sample.

\section{Scores on the five dimensions of the Greek NEO-PI-3}

The pattern of raw mean scores is similar to that seen in the US and elsewhere (Table 6).

As expected, Neuroticism was negatively related to the other factors, Extraversion was positively related to Openness, Agreeableness, and Conscientiousness, and Agreeableness was positively related to Conscientiousness. The links between Openness and Agreeableness or Conscientiousness were less evident but in the expected direction. Correlation between factors was never so high as to prevent discriminant validity.
Table 5 Factor loadings for Greek NEO-PI-3 facet scales after Procrustes rotation

\begin{tabular}{|c|c|c|c|c|c|c|}
\hline \multirow[b]{2}{*}{ NEO-PI-3 facet } & \multicolumn{5}{|c|}{ Factor } & \multirow[t]{2}{*}{ VCC } \\
\hline & $\mathbf{N}$ & $E$ & 0 & A & C & \\
\hline N1: Anxiety & .80 & -.08 & -.05 & .00 & -.02 & $.99^{\mathrm{a}}$ \\
\hline N2: Angry hostility & .68 & -.04 & -.13 & -.41 & -.06 & $.98^{\mathrm{a}}$ \\
\hline N3: Depression & .80 & -.24 & -.03 & .05 & -.15 & $.97^{\mathrm{a}}$ \\
\hline N4: Self-consciousness & .67 & -.16 & -.16 & .18 & -.06 & $.97^{\mathrm{a}}$ \\
\hline N5: Impulsiveness & .56 & .34 & .06 & -.35 & -.26 & $.98^{\mathrm{a}}$ \\
\hline N6: Vulnerability & .59 & -.24 & -.09 & -.08 & -.49 & $.96^{\mathrm{a}}$ \\
\hline E1: Warmth & -.16 & .61 & .11 & .47 & .21 & $.98^{\mathrm{a}}$ \\
\hline E2: Gregariousness & -.32 & .54 & .07 & .14 & .00 & $.96^{\mathrm{a}}$ \\
\hline E3: Assertiveness & -.34 & .38 & .07 & -.51 & .27 & $.94^{\mathrm{a}}$ \\
\hline E4: Activity & -.06 & .53 & -.03 & -.15 & .47 & $.94^{\mathrm{a}}$ \\
\hline E5: Excitement-seeking & .02 & .52 & .43 & -.23 & -.06 & $.87^{b}$ \\
\hline E6: Positive emotions & -.25 & .71 & .22 & .12 & -.05 & $.95^{\mathrm{a}}$ \\
\hline 01: Fantasy & .12 & .18 & .58 & -.14 & -.34 & $.99^{a}$ \\
\hline O2: Aesthetics & .14 & .08 & .76 & .12 & .09 & $.99^{\mathrm{a}}$ \\
\hline O3: Feelings & .28 & .44 & .51 & .04 & .22 & $.98^{\mathrm{a}}$ \\
\hline O4: Actions & -.23 & .08 & .53 & -.20 & -.07 & $.90^{\mathrm{b}}$ \\
\hline O5: Ideas & -.14 & .00 & .78 & -.01 & .15 & $.99^{\mathrm{a}}$ \\
\hline O6: Values & -.06 & .09 & .55 & -.02 & -.03 & $.96^{\mathrm{a}}$ \\
\hline A1: Trust & -.27 & .33 & -.05 & .51 & -.11 & $.92^{b}$ \\
\hline A2: Straightforwardness & -.06 & -.03 & -.04 & .64 & .19 & $.98^{\mathrm{a}}$ \\
\hline A3: Altruism & .02 & .34 & .06 & .59 & .46 & $.93^{b}$ \\
\hline A4: Compliance & -.23 & -.14 & -.07 & .73 & -.02 & $.99^{\mathrm{a}}$ \\
\hline A5: Modesty & .23 & -.09 & -.17 & .48 & .10 & $.95^{\mathrm{a}}$ \\
\hline A6: Tender-mindedness & .13 & .32 & .05 & .57 & .32 & $.88^{b}$ \\
\hline C1: Competence & -.29 & .25 & .07 & -.03 & .65 & $.98^{\mathrm{a}}$ \\
\hline C2: Order & .02 & -.08 & -.04 & .07 & .65 & $.95^{\mathrm{a}}$ \\
\hline C3: Dutifulness & .03 & .21 & .01 & .39 & .68 & $.90^{\mathrm{b}}$ \\
\hline C4: Achievement-striving & -.08 & .29 & .06 & -.05 & .75 & $.99^{a}$ \\
\hline C5: Self-discipline & -.29 & .07 & .00 & .15 & .74 & $.98^{a}$ \\
\hline C6: Deliberation & -.24 & -.30 & .00 & .25 & .66 & $.99^{\mathrm{a}}$ \\
\hline Congruence $^{c}$ & $.97^{\mathrm{a}}$ & $.96^{\mathrm{a}}$ & $.94^{\mathrm{a}}$ & $.96^{\mathrm{a}}$ & $.96^{\mathrm{a}}$ & $.96^{\mathrm{a}}$ \\
\hline
\end{tabular}

Note. $N=734$. These are principal components rotated to the American normative target (Costa and McCrae, [5]). Loadings greater than .40 in absolute magnitude are given in boldface type. Highest loadings are marked in italics. Abbreviations: NEO-PI-3 NEO Personality Inventory-3, N Neuroticism, E Extraversion, $O$ Openness, A Agreeableness, C Conscientiousness, VCC variable congruence coefficient.

${ }^{\mathrm{a} C}$ Congruence higher than that of $99 \%$ of rotations from random data; ${ }^{b}$ Congruence higher than that of $95 \%$ of rotations from random data;

${ }^{\mathrm{c}}$ Factor/total congruence coefficient with target matrix.

\section{Differences by gender, age, and education on the Greek NEO-PI-3}

Females scored higher than males on the Neuroticism and the Openness factors. Males scored marginally higher than females on the Conscientiousness factor (Table 7). 
Table 6 Mean values and correlations for the big five factors of the Greek NEO-PI-3

\begin{tabular}{|c|c|c|c|c|c|}
\hline & Mean $(95 \% \mathrm{Cl})$ & Neuroticism & Extraversion & Openness to experience & Agreeableness \\
\hline Neuroticism & 89.06 (87.64-90.48) & & & & \\
\hline Extraversion & $108.68(107.52-109.84)$ & $-0.546^{*}$ & & & \\
\hline Openness & $104.43(103.21-105.66)$ & $-0.108^{* *}$ & $0.466^{*}$ & & \\
\hline Agreeableness & 116.37 (115.23-117.52) & $-0.255^{*}$ & $0.545^{*}$ & $0.130^{* *}$ & \\
\hline Conscientiousness & $121.60(120.20-122.99)$ & $-0.506^{*}$ & $0.457^{*}$ & $0.113^{* *}$ & $0.635^{*}$ \\
\hline
\end{tabular}

${ }^{*} p<.0001 ;{ }^{* *} p<.05$.

Table 7 Differences by gender (females-males, after standardizing the scores as z-scores using the total samples $M$ and SD) and correlation with age and education on the Greek NEO-PI-3

\begin{tabular}{|c|c|c|c|}
\hline & \multirow[t]{2}{*}{$d$ sex } & \multicolumn{2}{|l|}{$r$} \\
\hline & & Age & Education \\
\hline N1: Anxiety & .57 & -.05 & -.01 \\
\hline N2: Angry hostility & .25 & .01 & -.01 \\
\hline N3: Depression & .39 & .02 & -.04 \\
\hline N4: Self-consciousness & .30 & .05 & -.12 \\
\hline N5: Impulsiveness & .30 & -.13 & .02 \\
\hline N6: Vulnerability & .52 & -.03 & -.03 \\
\hline E1: Warmth & -.06 & .05 & -.05 \\
\hline E2: Gregariousness & .13 & -.10 & -.01 \\
\hline E3: Assertiveness & -.29 & -.05 & .09 \\
\hline E4: Activity & -.01 & .04 & -.04 \\
\hline E5: Excitement-seeking & -.18 & -.30 & .09 \\
\hline E6: Positive emotions & .00 & -.17 & .03 \\
\hline O1: Fantasy & .14 & -.29 & .14 \\
\hline O2: Aesthetics & .20 & -.07 & .21 \\
\hline O3: Feelings & .26 & -.16 & .18 \\
\hline O4: Actions & .14 & -.23 & .16 \\
\hline O5: Ideas & .00 & -.17 & .23 \\
\hline O6: Values & .08 & -.24 & .26 \\
\hline A1: Trust & -.09 & .16 & -.02 \\
\hline A2: Straightforwardness & .14 & .08 & .01 \\
\hline A3: Altruism & -.01 & .11 & -.08 \\
\hline A4: Compliance & .06 & .20 & -.09 \\
\hline A5: Modesty & .03 & .15 & -.06 \\
\hline A6: Tender-mindedness & .03 & .09 & -.01 \\
\hline C1: Competence & -.31 & .07 & .05 \\
\hline C2: Order & .06 & .07 & -.02 \\
\hline C3: Dutifulness & .00 & .09 & .00 \\
\hline C4: Achievement-striving & -.21 & -.02 & .05 \\
\hline C5: Self-discipline & -.16 & .09 & -.01 \\
\hline C6: Deliberation & -.15 & .13 & -.01 \\
\hline
\end{tabular}

Overall, the pattern of gender differences is similar to what one sees around the world, except that Greek females did not score higher than males on Agreeableness.

Age and education were modestly related to Greek NEO-PI-3 facets.

\section{Discussion}

The current paper reports on the results of the Greek translation of the NEO-PI-3. Most facets exhibited Cronbach's alpha values above 0.60 , though overall, the internal consistency reliability measures of the Greek translation were lower than those observed in the original American sample. Confirmatory factor analysis failed to reach the predefined fit. However, it showed acceptable loading of the facets on their own hypothesized factors and very good estimations of Cronbach's alphas for these factors; therefore, it partly supports the five-factor structure of the NEO-PI-3. Principle components after Procrustes rotation closely resembled the factors of the American normative sample. Correlations between dimensions were as expected and similar to those reported in the literature.

The literature suggests that, overall, the psychometric properties of NEO-PI-R scales have been found to generalize across ages, cultures, and methods of measurement [7].

The internal consistency originally reported for both NEO-PI-R domains $(N=0.92, E=0.89, O=0.87, A=0.86$, $C=0.90)$ as well as facets $(0.56-0.81)$ was high. The internal consistency of the NEO-PI-3 was similar to that of the NEO-PI-R, with alphas ranging from 0.89-0.93 for domains and $0.54-0.83$ for facets [24,25]. The literature appears to support the internal consistencies listed in the manual. The Filipino translation of the NEO-PI-R has internal consistency of domain scores ranging from $0.78-0.90$ [26], with facet alphas having a median of 0.61 [27].

Test-retest reliability (administered 3 months later) of an early version of the NEO-PI domains was $N=0.87, E=$ 0.91 , and $O=0.86$ [28]. The test-retest reliability reported in the manual of the NEO-PI-R over 6 years was $N=0.83$, $E=0.82, \quad O=0.83, A=0.63$, and $C=0.79$. Costa and McCrae pointed out that this not only shows good reliability of the domains but also that they are stable over long periods of time (past the age of 30), as the scores 
more than 6 years apart were only marginally different from the scores measured a few months apart [5]. Other research has also shown acceptable test-retest reliability. A 2001 study by Kurtz and Parrish on the short-term testretest reliability yielded alpha coefficients $0.9-0.93$ for domains and $0.70-0.91$ for facets after a 1-week interval [29]. A 2006 study by Terracciano et al. [30] on long-term test-retest reliability yielded alpha coefficients $0.78-0.85$ for domains and $0.57-0.82$ for facets after a 10-year interval.

In terms of criterion validity, Conard (2006) found that Conscientiousness significantly predicted the GPA (grade point average) of college students, more so than by using Scholastic Assessment Test (SAT) scores alone [31]. Garcia et al. correlated a Spanish version of the NEO to predictors of teacher burnout in Sevilla, Spain. Neuroticism was related to the 'emotional exhaustion' factor of burnout with a correlation coefficient equal to 0.44 . Agreeableness related to the 'personal accomplishment' factor of burnout (which is negatively scored when predicting burnout) exhibited a score of $r=0.36$ [32]. A group of authors in 2006 found that in a minority students population, the Extraversion trait was correlated to Career Decision Making Self-Efficacy (CDMSE) with $r=0.30$, while Neuroticism was strongly related to Career Commitment after controlling for CDMSE $(r=0.42)$ [33]. Finally, in 2007, Korukonda reported that Neuroticism was positively related to computer anxiety, while Openness and Agreeableness were negatively related to each other [34].

Cross-cultural stability of an instrument can be considered evidence of its validity. A huge amount of crosscultural research has been carried out on the Five-Factor model of personality by utilizing the NEO-PI-R and its shorter version, the NEO-FFI. A collection of selected papers from various researchers across the globe have been presented covering various issues in cross-cultural research on the FFM [35]. This monograph has also presented data for the FFM from several cultures. The robustness of the FFM has been proven across different cultures; these include but are not limited to the following: Chinese [36,37], Estonian and Finnish [38], Filipino and French [39], Indian [40], Portuguese [41], Russian [42], South Korean [43], Turkish [44], Vietnamese [45], sub-Saharan cultures like Zimbabwean [46], Austrian, former East and West German, and Switzerland's culture [47]. On the basis of the data from 16 cultures, it has been suggested that the concepts of Neuroticism, Openness, and Conscientiousness are cross-culturally valid, while Extraversion and Agreeableness are components of interpersonal circumflex and are more sensitive to cultural context [48]. It is interesting to note that in the Zuckerman five-factor model 'Openness to experience' is deliberately excluded because Zuckerman suggested that it does not meet the criteria for a truly 'basic' factor of personality [49]. Furthermore, it seems that the age differences in the five factors of personality across the adult life span are paralleled in samples from Germany, Italy, Portugal, Croatia, and South Korea [50]. The age and gender differences and fluctuations found in the original American sample [3] were generally confirmed in an analysis of the data from 51 cultures [51-53]. These findings are paralleled by the results of the current Greek validation study.

In conclusion, we submit that the results of the current study confirm the reliability of the Greek translation and adaptation of the NEO-PI-3. The inventory has comparable psychometric properties in its Greek version as in the original and other national versions, although with somewhat lower values, and it is suitable for clinical as well as research use in Greek speaking populations.

\section{Competing interests}

The authors declare that they have no competing interests.

\section{Authors' contributions}

KNF and HA conceived of the study and participated in its design and coordination, performed the statistical analysis, helped in the gathering of data the analysis and interpretation of data, and participated in the authoring of the manuscript. MS and SM helped in the gathering of data the analysis and interpretation of data and participated in the authoring of the manuscript. EP $\mathrm{KM}, \mathrm{PP}, \mathrm{MK}, \mathrm{EM}, \mathrm{EL}$, and $\mathrm{EB}$ helped in the gathering and interpretation of data and participated in the authoring of the manuscript. $A P, L T, X G, N M$, and KA participated in its design and coordination and in the analysis and interpretation of data and participated in the authoring of the manuscript. PC and RM participated in its design and coordination and in the analysis and interpretation of data and participated in the authoring of the manuscript. All authors read and approved the final manuscript.

\section{Author details}

${ }^{1}$ Third Department of Psychiatry, School of Medicine, Aristotle University of Thessaloniki, Thessaloniki, Greece. ${ }^{2}$ Research associate Aristotle University of Thessaloniki, Thessaloniki, Greece. ${ }^{3}$ Department of Psychiatry, 424 Military Hospital, Thessaloniki, Greece. ${ }^{4}$ Mental Health Hospital of Thessaloniki, Thessaloniki, Greece. ${ }^{5}$ Psychologist in private practice, Athens, Greece. ${ }^{6}$ Center of Liaison Psychiatry and Psychosomatics, University Hospital, University of Cagliari, Italy, and Centro Medico Genneruxi, Cagliari, Italy. ${ }^{7}$ Mood Center LucioBini, Cagliari and Rome, Italy. ${ }^{8}$ McLean Hospital, Harvard Medical School, Boston, USA. 'Department of Clinical and Theoretical Mental Health, Faculty of Medicine, Semmelweis University, Budapest, Hungary. ${ }^{10}$ Department of Psychiatry, University of California, San Diego, California, USA. ${ }^{11}$ Studies of Temperament and Creativity, Paris, France. ${ }^{12}$ International Mood Center, University of California, San Diego, CA, USA. ${ }^{13}$ Laboratory of Behavioral Neuroscience Biomedical Research Center, Baltimore, MD, USA. ${ }^{14}$ Baltimore, MD, USA.

\section{Received: 23 September 2014 Accepted: 11 November 2014} 2.

\section{References}

1. Shock NW, Greulich RC, Andres R, Arenberg D, Costa PT, Lakatta EG: Normal Human Aging: The Baltimore Longitudinal Study of Aging. Bethesda, MD: National Institutes of Health; 1984.

2. Costa PT, McCrae RR: The NEO Personality Inventory Manual. Odessa, FL: Psychological Assessment Resources; 1985.

3. McCrae RR, Martin TA, Costa PT Jr: Age trends and age norms for the NEO Personality Inventory-3 in adolescents and adults. Assessment 2005, 12(4):363-373. 
4. Katki H: Invited commentary: evidence-based evaluation of $p$ values and Bayes factors. Am J Epidemiology 2008, 168(4):384-388.

5. Costa PT, McCrae RR: NEO PI-R Professional Manual. Odessa, FL: Psychological Assessment Resources, Inc.; 1992

6. Nunnally JC: Psychometric Theory. 2nd edition. New York, NY: McGraw-Hill Education; 1978

7. McCrae RR, Kurtz JE, Yamagata S, Terracciano A: Internal consistency, retest reliability, and their implications for personality scale validity. Pers Soc Psychol Rev 2011, 15(1):28-50.

8. Cohen J: Statistical Power Analysis for the Behavioral Sciences. 2nd edition. Hillsdale, NJ: Taylor \& Francis; 1988.

9. Rosseel Y: Lavaan: an R package for structural equation modeling. J Stat Softw 2012, 48(2):1-36.

10. R Development Core Team: R: A Language and Environment for Statistical Computing. Vienna, Austria: the R Foundation for Statistical Computing; 2011. Available online at http://www.R-project.org/. ISBN 3-900051-07-0.

11. Narayanan A: A review of eight software packages for structural equation modelling. Am Statistican 2012, 66:129-138.

12. Curran PJ, West SG, Finch JF: The robustness of test statistics to nonnormality and specification error in confirmatory factor analysis. Psychol Methods 1996, 1:16-29.

13. Hu L, Bentler PM: Cutoff criteria for fit indices in covariance structure analysis: conventional criteria versus new alternatives. Struct Equ Model 1999, 6:1-55.

14. Tanaka JS: Multifaceted conceptions of fit in structural equation models In Testing Structural Equation Models. Edited by Bollen KA, Long JS. Newbury Park, CA: Sage; 1993:10-39.

15. Byrne BM: A Primer of LISREL: Basic Applications and Programming for Confirmatory Factor Analytic Model. New York, NY: Springer-Verlag; 1989.

16. Browne MW, Cudeck R: Alternative ways of assessing model fit. In Testing Structural Equation Models. Edited by Bollen KA, Long JS. Newbury Park, CA: Sage; 1993:136-161.

17. Herrmann A, Pfister HR: Simple measures and complex structures: is it worth employing a more complex model of personality in Big Five inventories? J Res Pers 2013, 47:599-608.

18. McCrae RR, Zonderman AB, Costa PT, Bond MH, Paunonen SV: Evaluating replicability of factors in the revised NEO Personality Inventory: confirmatory factor analysis versus Procrustes rotation. J Pers Soc Psychol 1996, 70:552-566.

19. Terracciano A: The Italian version of the NEO PI-R: conceptual and empirical support for the use of targeted rotation. Pers Individ Dif 2003, 35(8):1859-1872

20. Aluja A, Garcia O, Garcia LF, Seisdedos N: Invariance of the "NEO-PI-R" factor structure across exploratory and confirmatory y factor analyses. Personal Individ Differ 2005, 38:1879-1889.

21. Comrey AL, Lee HB: A First Course in Factor Analysis. 2nd edition. Hillsdale, NJ: Erlbaum; 1992

22. Tucker LR: A Method for the Synthesis of Factor Analysis Studies (Personnel Research Section Report No. 984). Washington: Department of the Army; 1951.

23. Sakamoto S, Kijima N, Tomoda A, Kambara M: Factor structures of the Zung self-rating depression scale (SDS) for undergraduates. J Clin Psychol 1998, 54(4):477-487.

24. McCrae RR, Costa PT: NEO Inventories: Professional Manual. Lutz, FL: Psychological Assessment Resources, Inc.; 2010.

25. De Fruyt F, De Bolle M, McCrae RR, Terracciano A, Costa PT Jr, Collaborator of the Adolescent Personality Profiles of Cultures P: Assessing the universal structure of personality in early adolescence: the NEO-PI-R and NEO-PI-3 in 24 cultures. Assessment 2009, 16(3):301-311.

26. Church AT, Katigbak MS: Indigenization of psychology in the Philippines. Int J Psychol 2002, 37(3):129-148.

27. Katigbak MS, Church AT, Guanzon-Lapena MA, Carlota AJ, Del P: Are indigenous personality dimensions culture specific? Philippine inventories and the five-factor model. J Pers Soc Psychol 2002, 82(1):89-101.

28. McCrae RR, Costa PT: Joint factors in self-reports and ratings: neuroticism, extraversion and openness to experience. Personal Individ Differ 1983, 4(3):245-255.

29. Kurtz JE, Parrish CL: Semantic response consistency and protocol validity in structured personality assessment: the case of the NEO PI-R. J Pers Assess 2001, 76:315-332.

30. Terracciano A, Costa PT Jr, McCrae RR: Personality plasticity after age $\mathbf{3 0 .}$ Pers Soc Psychol Bull 2006, 32(8):999-1009.
31. Conard MA: Aptitude is not enough: how personality and behavior predict academic performance. J Res Pers 2006, 40(3):339-346.

32. García FJC, Muñoz EMP, Ortiz MÁC: Personality and contextual variables in teacher burnout. Personal Individ Differ 2005, 38(4):929-940.

33. Wang N, Jome LM, Haase RF, Bruch MA: The role of personality and career decision-making self-efficacy in the career choice commitment of college students. J Career Assess 2006, 14(3):312-332.

34. Korukonda AR: Differences that do matter: a dialectic analysis of individual characteristics and personality dimensions contributing to computer anxiety. Comput Hum Behav 2007, 23(4):1921-1942.

35. McCrae RR, Allik J: The Five-Factor Model of Personality Across Cultures. New York, N.Y: Kluwer AcademicPublisher; 2002

36. McCrae RR, Costa PT, Yik MSM: Universal Aspects of Chinese Personality Structure. Hong Kong: Oxford University Press; 1996

37. Yik MSM, Bond MH: Exploring the dimensions of Chinese person perception with indigenous and imported constructs: creating a culturally balanced scale. Int J Psychol 1993, 28:75-95.

38. Pulver A, Allik J, Pulkkinen L, Hämäläinen M: The Big Five personality inventory in two non-Indo-European languages. Eur J Personal 1995, 9(2):109-124.

39. McCrae RR, Costa PT, Pilar GH, Rolland JP, Parker WD: Cross-cultural assessment of the five-factor model: the revised NEO Personality Inventory. J Cross-Cult Psychol 1998, 29:171-188.

40. Lodhi PH, Deo S, Belhekar VM: The Five-Factor Model of Personality in Indian Context: Measurement and Correlates. New York, N.Y: Kluwer AcademicPublisher; 2002

41. Lima MP: Personality and Culture: The Portuguese Case. New York, N.Y: Kluwer Academic Publisher; 2002.

42. Martin TA, Oryol VE, Rukavishnikov AA, Senin IG: Applications of the Russian NEO-PI-R. In XXVIIth International Congress of Psychology. Stockholm, Sweden: National Academies; 2000.

43. Piedmont RL, Chae $\mathrm{JH}$ : Cross-cultural generalizability of the five-factor model of personality: development and validation of the NEO-PI-R for Koreans. J Cross-Cult Psychol 1997, 28(2):131-155.

44. Gülgöz S: Five-Factor Model and NEO-PI-R in Turkey. New York, N.Y: Kluwer Academic Publisher; 2002.

45. Leininger A: Vietnamese American Personality and Acculturation: An Exploration Between Personality Traits and Cultural Goals. New York, N.Y: Kluwer Academic Publisher; 2002.

46. Piedmont RL, Bain E, McCrae RR, Costa PT: The Applicability of Five Factor Model in sub Saharan Culture: The NEO-PI-R in Shona. New York, N.Y: Kluwer Academic Publisher; 2002.

47. Angleitner A, Ostendorf F: The FFM: a comparison of German speaking countries (Austria, Former East and West Germany, and Switzerland). In XXVIIth International Congress of Psychology. Stockholm, Sweden: National Academies; 2000.

48. Rolland JP: Cross-Cultural Validity of the Five Factor Model of Personality. In XXVIIth International Congress of Psychology. Stockholm, Sweden: National Academies; 2000.

49. Zuckerman M: Zuckerman-Kuhlman Personality Questionnaire (ZKPQ): an Alternative Five-Factorial Model. Seattle, WA: Hogrefe \& Huber; 2002

50. McCrae RR, Costa PT Jr, Pedroso De Lima M, Simoes A, Ostendorf F, Angleitner A, Marusic I, Bratko D, Caprara GV, Barbaranelli C, Chae JH, Piedmont RL: Age differences in personality across the adult life span: parallels in five cultures. Dev Psychol 1999, 35(2):466-477.

51. McCrae RR: Trait psychology and culture: exploring intercultural comparisons. J Pers 2001, 69(6):819-846.

52. McCrae RR: NEO-PI-R Data from 36 Cultures: Further Intercultural Comparisons. New York, NY: Kluwer Academic Publisher; 2002.

53. McCrae RR, Terracciano A: Personality profiles of cultures: aggregate personality traits. J Pers Soc Psychol 2005, 89(3):407-425.

doi:10.1186/s12991-014-0036-9

Cite this article as: Fountoulakis et al: Standardization of the NEO-PI-3 in the Greek general population. Annals of General Psychiatry 2014 13:36. 